\title{
New method for determining the optimum fluid temperature when heating pressure thick-walled components with openings
}

\author{
Dawid Taler ${ }^{1, *}$, Piotr Dzierwa ${ }^{1}$, and Jan Taler ${ }^{1}$ \\ ${ }^{1}$ Faculty of Environmental and Energy Engineering, Cracow University of Technology, ul. Warszawska 24, Cracow, Poland
}

\begin{abstract}
A new approximate method of optimum heating cylindrical pressure elements weakened by openings was proposed. Optimum variations in fluid temperature when heating the pressure component were determined from the condition that the total circumferential stress at the edge of the opening, resulting from the thermal load and pressure is equal to the allowable stress. The allowable stress is determined from the Wöhler fatigue diagram for a given number of start-ups and shutdowns of a power unit from the cold state. Optimum temperature changes are difficult to estimate at the beginning of the heating, using both exact analytical and numerical methods. In case of analytical methods, this is due to the very slow convergence of a series for near-zero time in the exact solution. In this paper, the optimum temperature changes of the fluid at the beginning of heating were determined using the heat balance integral method (HBIM). This method makes it possible to determine with high accuracy the temperature of the fluid for times close to zero, i.e., at the beginning of the heating process. In the second stage of heating, the optimum fluid temperature was determined on the assumption of a quasi-steady temperature field in the pressure element.
\end{abstract}

\section{Introduction}

Due to the rapid development of wind farms for electricity production, which are characterized by high power variability over time, it is necessary to build steam-gas systems [1-2] in the power system and the need to increase the flexibility of conventional steam units to ensure a balance between supply and demand for electricity. Two different dynamic models were developed and compared, for simulating combined cycle power plants in order to predict the remnant life of the power plant equipment, as a function of the past and the forecasted operation strategy [1]. Transient mathematical model of the drum was developed in [2]. Performance studies over wide-range dynamic operation of the drum were conducted. During frequent start-ups and shutdowns of large power boilers, it is necessary to determine the permissible heating and cooling rates of the so-called critical elements of the power unit for which the permissible rates of temperature changes are the lowest [3-5]. Critical elements are usually monitored to ensure safe operation in fast start-up conditions. The sum of the circumferential thermal and pressure stresses shall not exceed the stresses permitted due to low cycle fatigue. Methods for monitoring temperature and thermal stresses in pressure elements were developed in the works [3-5]. Based on the measurement of transient temperature histories on the external thermally insulated surface of the pressure element, transient temperature, and stress distributions can be reconstructed without the need to determine the heat transfer coefficient on the internal surface of the element. In the paper [4], a new method of temperature measurement was proposed. Thanks to the new thermometer, it is possible to measure fast, transient temperature changes with high accuracy and to determine the value of heat transfer coefficient on the inner surface of the pressure element. Using the measured fluid temperature and estimated heat transfer coefficient, the thermal stress distribution can be determined in components with complicated geometry. The heating and cooling time of critical elements of the boiler can be shortened by determining the time optimum temperature variations of the fluid [6-7]. An optimum temperature of the fluid was determined in work [6] so that the temperature of the wall at the point lying inside of it changes linearly in the function of time. Taler et al. [7] proposed an optimization of the boiler start-up so that the stress at the edge of the opening at the concentration point does not exceed the permissible stress. The fuel mass flow rate supplied to the furnace chamber of the boiler was determined to ensure the shortest time for starting the steam boiler while maintaining the condition that the stresses at the edges of the openings in the drum do not exceed the permissible stresses. In the article [8], the optimum temperature of the water supplied to the boiler evaporator was determined when it is flooded with hot water at the beginning of the boiler start-up.

In this paper, the new method of determining the fluid temperature during optimum heating of the thick-walled cylindrical boiler elements is presented. The advantage of this method is simple relationships for calculating the

\footnotetext{
* Corresponding author: jtaler@mech.pk.edu.pl
} 
step change of the fluid temperature at the beginning of the heating process. The proposed method can be easily applied in practice.

\section{Mathematical formulation}

Circumferential stress at the opening edge at a point in the plane of the cross-section (Fig. 1), which is the sum of the thermal stress and the stress due to pressure, is given by the following formula

$$
\sigma_{\varphi, P_{1}}=\alpha_{T} \sigma_{T}+\alpha_{p} \sigma_{p}
$$

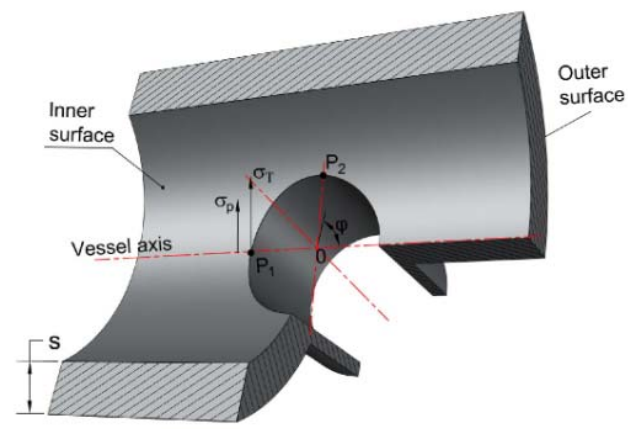

Fig. 1. Circumferential thermal stress and stress due to pressure on the edge of the opening in the cylindrical pressure component.

The thermal stress $\sigma_{T}$ and stress due to pressure $\sigma_{p}$ are determined by the following relationships

$$
\begin{gathered}
\sigma_{T}=\alpha_{T} \frac{E \beta}{1-v}\left[T_{m}(t)-T\left(\rho_{i n}, t\right)\right] \\
\sigma_{p}=\alpha_{p} \frac{\left(p-p_{a}\right) d_{m}}{2 s}
\end{gathered}
$$

where: $d_{m}=\left(d_{i n}+d_{o}\right) / 2=r_{i n}+r_{o}$ - mean diameter of the pressure component, $s=r_{o}-r_{i n}$ - wall thickness.

The symbol $T_{m}(t)$ in Eq. (3) indicates the mean temperature of the cylindrical component defined as

$$
T_{m}=\frac{2}{1-\rho_{\text {in }}^{2}} \int_{\rho_{\text {in }}}^{1} \rho T d \rho
$$

During optimum pressure heating of the thick-walled component, the total circumferential stress at $P_{1}$ shall be less than or equal to the allowable stress due to low cycle fatigue

$$
\sigma_{\varphi, P_{1}} \leq \sigma_{a l}
$$

The condition (5) may be transformed into the following form

$$
\frac{2}{1-\rho_{\text {in }}^{2}} \int_{\rho_{\text {in }}}^{1} \rho T(\rho, t) d \rho-T\left(\rho_{\text {in }}, t\right) \leq \sigma_{a l}^{*}
$$

The dimensionless allowable stress $\sigma_{a l}^{*}$ is given by the following formula

$$
\begin{aligned}
\sigma_{a l}^{*} & =\left[\sigma_{a l}-\alpha_{p} \frac{\left(p-p_{a}\right) d_{m}}{2 s}\right] \frac{1-v}{\alpha_{T} E \beta}= \\
& =b_{1}-c_{1} T(p, T)
\end{aligned}
$$

where $b_{1}$ and $c_{1}$ are the constants.

The transient temperature field in a cylindrical pressure element is determined from the heat conduction equation

$$
\frac{\partial T}{\partial F_{O}}=\frac{1}{\rho} \frac{\partial}{\partial \rho}\left(\rho \frac{\partial T}{\partial \rho}\right)
$$

where: $\rho=r / r_{o}$ - dimensionless radius, $F o=\kappa t / r_{o}^{2}$ Fourier number, $\kappa=k_{m} / c_{m} \rho_{m}$ - thermal diffusivity of the component material.

Equation (8) is subject to the following boundary condition on the outer surface of the cylinder

$$
\left.\frac{\partial T}{\partial \rho}\right|_{\rho=1}=0
$$

The boundary condition (9) means that the outer surface of the pressure element is thermally insulated. The heat conduction equation (8) must also satisfy the condition (5), i.e., the circumferential stress on the internal surface of the component during optimum heating is equal to the permissible stress. It must be noted that the thermal longitudinal stress on the inner surface of the cylindrical component is equal to the circumferential stress, assuming that the ends of the component can freely elongate.

The initial temperature of the element is uniform and equal to $T_{0}$, i.e., the initial condition is

$$
\left.T\right|_{t=0}=T_{0}
$$

By transforming the convective boundary condition on the internal surface, the following relationship is obtained for the calculation of the fluid temperature

$$
T_{f}(t)=T\left(r_{i n}, t\right)-\left.\frac{1}{B i} \frac{\partial T}{\partial \rho}\right|_{\rho=\rho_{\text {in }}}
$$

where $B i=h r_{o} / k_{m}$ stands for the Biot number.

After the determination of the transient temperature distribution inside the cylindrical element from the initial-boundary problem (6), (8), and (9) the fluid temperature $T_{f}(t)$ is then determined using Eq. (11).

The analysis of works concerning the optimum heating of thick-walled boiler elements shows that it is difficult to determine the optimum fluid temperature changes in the initial phase of heating. This is due to the slow convergence of the power series in the analytical 
solutions needed to solve the integral equation from which the optimum fluid temperature is determined. When solving the integral equation, the so-called influence function is used, which is the solution of the linear heat conduction equation at a stepwise unit variation of fluid temperature. At the beginning of heating, when the time $t$ is close to zero, the convergence of the power series is very slow. Even with a very large number of terms in the power series, the accuracy of the analytical solution is not satisfactory. Another major disadvantage when determining the influence function using the analytical formula is the need to determine a large number of roots of the characteristic equation [5]. In this paper, the heat balance integral method (HBIM) [9-10] was applied to determine the optimum fluid temperature in the initial phase of heating the element. This method uses the concept of heat penetration depth, which is similar to the thickness of the thermal boundary layer in the fluid mechanics proposed by Prandtl [11]. This method is widely used in the analysis of solid melting. The HBIM was used to determine the temperature field in the thick-walled element in the initial phase of optimum heating when the heat penetration depth is less than or equal to the wall thickness. Theoretically determined temperature variation of the fluid is difficult to achieve in practice because the permissible fluid temperature at the beginning of heating is very high. During heating, the temperature of the fluid very quickly decreases to a certain minimum after reaching, which starts to increase. The minimum fluid temperature occurs at the end of the first phase of heating the element when the depth of heat penetration is equal to the wall thickness. In practice, theoretically determined initial changes in fluid temperature were replaced by a step change in fluid temperature from the initial temperature to the minimum temperature of the fluid obtained with HBIM.

Further optimum fluid temperature changes were determined on the assumption that the temperature field in the cylindrical element is quasi-steady. Optimum fluid temperature variations over time were determined for the drum - downcomer junction. The temperature and stress distribution in a connection of the thick-walled drum with the downcomer tube was determined using the method proposed in the paper. The finite element method (FEM) was used to verify that the total circumferential stress at the point $P_{1}$ does not exceed the permissible stress when the fluid temperature is timeoptimum according to the procedure proposed in the paper.

\section{Optimum temperature changes of the fluid in the initial phase of heating the cylindrical element}

Optimum fluid temperature changes are determined using HBIM. The integration of transient heat conduction equation (8) over a dimensionless radius within the interval from $\rho_{i n}$ to $\rho_{i n}+\delta^{*}$, where $\delta^{*}=\delta / r_{o}$ is the dimensionless depth of heat penetration, gives

$$
\int_{\rho_{\text {in }}}^{\rho_{\text {in }}+\delta^{*}} \frac{\partial T}{\partial F_{O}} \rho d \rho=\int_{\rho_{\text {in }}}^{\rho_{\text {in }}+\delta^{*}} \frac{1}{\rho} \frac{\partial}{\partial \rho}\left(\rho \frac{\partial T}{\partial \rho}\right) \rho d \rho
$$

Equation (12) was transformed using the Leibniz rule [12] to the following form

$$
\begin{aligned}
& \frac{d}{d F_{O}} \int_{\rho_{\text {in }}}^{\rho_{\text {in }}+\delta^{*}} \rho\left(T-T_{0}\right) d \rho= \\
& \left.\left(\rho_{\text {in }}+\delta^{*}\right) \frac{\partial T}{\partial \rho}\right|_{\rho=\rho_{\text {in }}+\delta^{*}}-\left.\rho_{\text {in }} \frac{\partial T}{\partial \rho}\right|_{\rho=\rho_{\text {in }}}
\end{aligned}
$$

The temperature distribution in the first heat penetration phase was approximated using the polynomial of the second degree (Fig. 2)

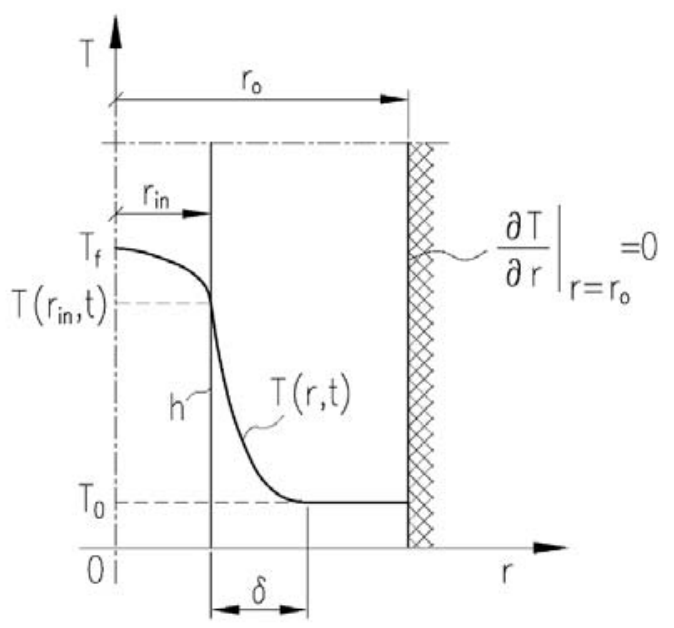

Fig. 2. Diagram illustrating the temperature distribution in the

pressure element and the depth of heat penetration.

$$
\begin{gathered}
T=A+B \rho+C \rho^{2} \quad \rho_{\text {in }} \leq \rho \leq\left(\rho_{\text {in }}+\delta^{*}\right) \\
T=T_{0} \quad\left(\rho_{\text {in }}+\delta^{*}\right) \leq \rho \leq 1
\end{gathered}
$$

The constants $A, B$, and $C$ were determined from condition (6) and the boundary conditions on the radius $\left(\rho_{\text {in }}+\delta^{*}\right)$

$$
\begin{aligned}
& \left.T\right|_{\rho=\rho_{i n}+\delta^{*}}=T_{0} \\
& \left.\frac{\partial T}{\partial \rho}\right|_{\rho=\rho_{\text {in }}+\delta^{*}}=0
\end{aligned}
$$

Substituting the constants $A, B$ and $C$ into (14) gives

$$
T=D\left(1-\frac{\rho-\rho_{i n}}{\delta^{*}}\right)^{2}+T_{0}, \quad \rho_{i n} \leq \rho \leq\left(\rho_{i n}+\delta^{*}\right)
$$

where the constant $D$ is given by 


$$
D=\frac{6\left(1-\rho_{i n}^{2}\right) b_{1}}{\left(\delta^{*}\right)^{2}+4 \rho_{i n} \delta^{*}-6\left(c_{1}-1\right)\left(1-\rho_{\text {in }}^{2}\right)}
$$

The first term in the energy balance equation (13) is equal to zero due to the boundary condition (17). After substituting the temperature distribution given by the expression (18) into the heat balance equation (13), the following first order ordinary differential equation for dimensionless heat penetration depth is obtained after the transformations

$$
\begin{aligned}
& \frac{\left(\delta^{*}\right)^{2}+2 \rho_{\text {in }} \delta^{*}}{\left(\delta^{*}\right)^{2}+4 \rho_{i n} \delta^{*}-6\left(c_{1}-1\right)\left(1-\rho_{\text {in }}^{2}\right)} \frac{d \delta^{*}}{d F o}= \\
& =\frac{2 \rho_{\text {in }}}{\left(c_{1}-1\right)\left(\rho_{i n}^{2}-1\right)}
\end{aligned}
$$

Equation (20) was solved with the following initial condition

$$
\left.\delta^{*}\right|_{F o=0}=0
$$

Using the table of indefinite integrals [13], the following solution of the differential equation (20) with the initial condition (21) was obtained

$$
\begin{aligned}
& F O=\frac{\left(c_{1}-1\right)\left(1-\rho_{i n}^{2}\right)}{2 \rho_{i n}} \times \\
& \left\{\delta^{*}-\rho_{i n} \ln \frac{\left(\delta^{*}\right)^{2}+4 \rho_{i n} \delta^{*}+6\left(c_{1}-1\right)\left(1-\rho_{i n}^{2}\right)}{6\left(c_{1}-1\right)\left(1-\rho_{i n}^{2}\right)}+\right. \\
& \frac{4 \rho_{i n}^{2}-6\left(c_{1}-1\right)\left(1-\rho_{i n}^{2}\right)}{\sqrt{\Delta}} \times \\
& \left.\times \ln \frac{\left(2 \delta^{*}+4 \rho_{\text {in }}-\sqrt{\Delta}\right)\left(4 \rho_{i n}+\sqrt{\Delta}\right)}{\left(2 \delta^{*}+4 \rho_{i n}+\sqrt{\Delta}\right)\left(4 \rho_{i n}-\sqrt{\Delta}\right)}\right\}
\end{aligned}
$$

where

$$
\Delta=16 \rho_{i n}^{2}-24\left(c_{1}-1\right)\left(1-p_{2}\right)
$$

Equation (20) with the condition (21) was also solved using the fourth order Runge-Kutta method, obtaining the same results as from the analytical formula (22). From the convective boundary condition on the inner surface

$$
-\left.\frac{\partial T}{\partial \rho}\right|_{\rho=\rho_{\text {in }}}=B i\left[T_{f}-\left.T\right|_{\rho=\rho_{\text {in }}}\right]
$$

the optimum fluid temperature is obtained

$$
T_{f}(t)=D\left(1+\frac{2}{B i \delta^{*}}\right)+T_{0}
$$

The Bi symbol in Eqs.(24)-(25) stands for the Biot number defined as follows

$$
B i=h r_{o} / k
$$

When the depth of heat penetration $\delta$ is equal to the wall thickness $s$ of the component, the second stage of heating begins. The fluid temperature rises from the minimum value to the nominal temperature during this period.

\section{The second stage of optimum heating of the pressure element}

After the first heat penetration phase is completed, the fluid temperature is the lowest.

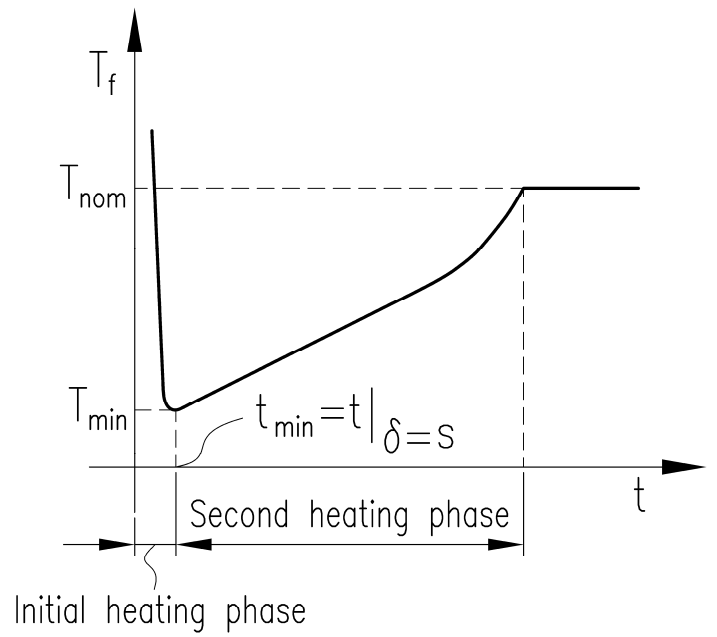

Fig. 3. Optimum fluid temperature in the first and second phase

of heating the pressure element.

The minimum $T_{\min }$ temperature is taken as the initial temperature in the second phase of the pressure element heating. The initial condition for the fluid temperature in the second phase is as follows (Fig. 3)

$$
\left.T_{f}\right|_{\delta^{*}=s / r_{o}}=T_{\min }
$$

In the second phase, the allowable heating rate of the pressure element is determined from the condition (5) which for the quasi-steady state temperature field takes the form

$$
\begin{aligned}
& \alpha_{p} \frac{\left(p-p_{a}\right) d_{m}}{2 s}+ \\
& \alpha_{T} \frac{E \beta}{1-v} \frac{\left(u^{2}-1\right)\left(3 u^{2}-1\right)-4 u^{4} \ln u}{8\left(u^{2}-1\right)(u-1)^{2}} \frac{v_{T} s^{2}}{\kappa} \leq \sigma_{a l}
\end{aligned}
$$

where: $u=r_{o} / r_{i n}$ - the ratio of the outer to the inner radius.

The allowable heating rate $v_{T 1}$ for the pressure $p_{1}$ at the beginning of the boiler start-up and the allowable heating rate $v_{T 2}$ for the pressure $p_{2}$ at the end of the boiler start-up were determined using condition (28). The permissible heating rate for any pressure within $p_{1} \leq p \leq p_{2}$ is determined from linear interpolation [14] 
between $v_{T 1}$ and $v_{T 2}$ velocities determined for $p_{1}$ and $p_{2}$ respectively (Fig. 4 )

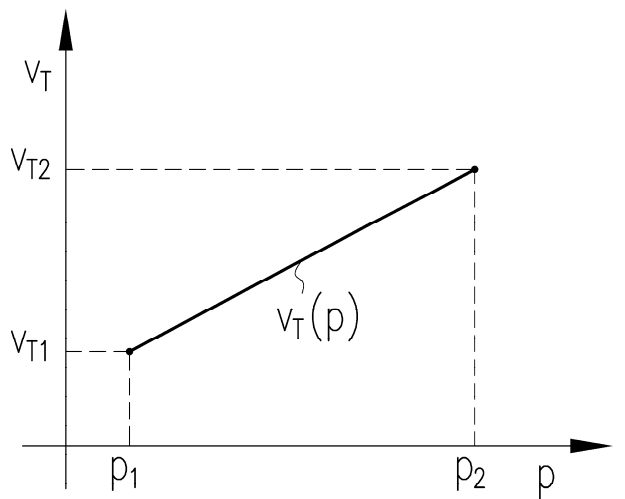

Fig. 3. Heating rate $v_{T}$ of a pressure element as a linear function of pressure $p$.

$$
v_{T}(p)=\frac{p_{2} v_{T 1}-p_{1} v_{T 2}}{p_{2}-p_{1}}+\frac{v_{T 2}-v_{T 1}}{p_{2}-p_{1}} p
$$

The temperature of the fluid is determined by integrating the following differential equation

$$
\frac{d T_{f}}{d t}=v_{T}(p)
$$

which is subject to the initial condition (27).

Equation (30) has been integrated using the Runge-Kutta method of the fourth order. When the nominal fluid temperature $T_{\text {nom }}$ is reached, the fluid temperature remains constant and equals $T_{\text {nom }}$ (Fig. 3 ). In the case of superheated steam, pressure and temperature are independent of each other. If the steam is saturated, its temperature is a function of the pressure. For saturated water steam, the steam pressure was approximated as a function of temperature. The values of pressure for the least square approximation were taken from international steam tables [15].

\section{Optimum heating of the drum- downcomer junction}

The optimum temperature changes of the fluid during the heating of the boiler drum were determined. The boiler with natural circulation is fired with hard coal. The electrical power of the steam unit under analysis is 200 MW. The outer diameter of the drum and its thickness are respectively $d_{o}=2 r_{o}=1.88 \mathrm{~m}$ and $s=0.085 \mathrm{~m}$. The outer diameter of the downcomer is $d_{d}=0.159 \mathrm{~m}$. The thickness of the downcomer wall is $s_{d}=0.016 \mathrm{~m}$. The drum is made of 18CuNMT low alloy steel with the following chemical composition: $\mathrm{C}-0.15 \div 0.20 \%, \mathrm{Mn}-$ $0.8 \%, \mathrm{Si}-0.30 \div 0.50 \%, \mathrm{P}-0.04 \%, \mathrm{~S}-0.04 \%$, Ni $1 \div 1.2 \%$, Cr $-<0.3 \%$, Cu $-1 \div 1.2 \%$, Ti $-0.03 \div 0.05 \%$, Mo $-0.25 \div 0.32 \%$. The following thermo-physical properties of the drum and downcomer material were assumed for calculations: thermal conductivity $k_{m}=41.9 \mathrm{~W} /(\mathrm{m} \cdot \mathrm{K}), \quad$ specific heat capacity $c_{m}=468 \mathrm{~J} /(\mathrm{kg} \cdot \mathrm{K}), \quad$ density $\rho_{m}=7800 \mathrm{~kg} / \mathrm{m}^{3}$, Young modulus $E=181 \cdot 10^{5} \mathrm{MPa}$, linear thermal expansion coefficient $\beta=1.19 \cdot 10^{-5} 1 / \mathrm{K}$, Poisson's ratio $v=0.292$. The heat transfer coefficient on the inner surface of the drum and downcomer was $h=500 \mathrm{~W} /\left(\mathrm{m}^{2} \cdot \mathrm{K}\right)$.

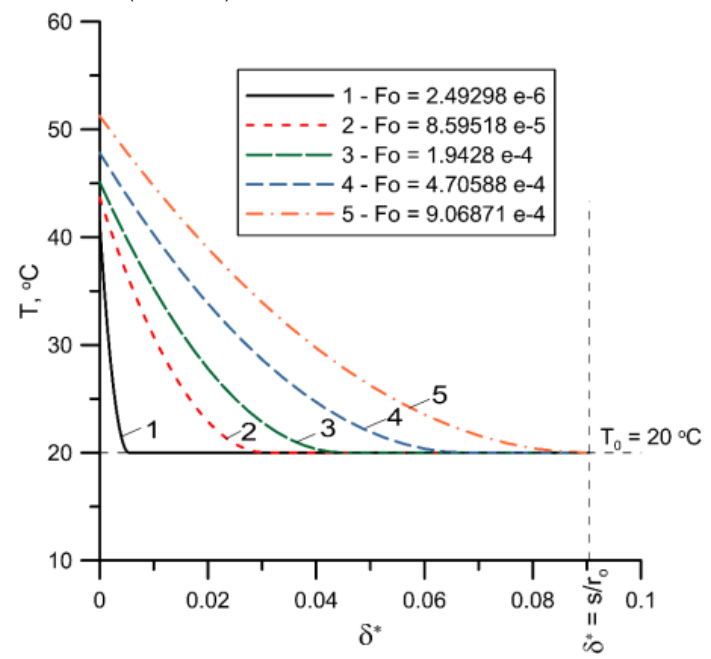

Fig. 5. The distribution of the temperature in the wall of the cylindrical element at selected time points during the first heating phase.

The allowable stress at the opening edge equal to $\sigma_{a l}=-91.4 \mathrm{MPa}$ were determined in accordance with the European standard EN 12952-3 [23]. The constant $c_{1}$ in Eq. (7) was assumed to be zero.

The stress concentration factors of circumferential thermal and pressure stresses at the point $P_{1}$ were determined by Finite Element Method (FEM) using the program ANSYS. These coefficients were as follows: $\alpha_{p}=2.67$ and $\alpha_{T}=2.22$. The distribution of the temperature in the wall of the cylindrical element at selected time points of time during the first heating phase is shown in Fig. 5.

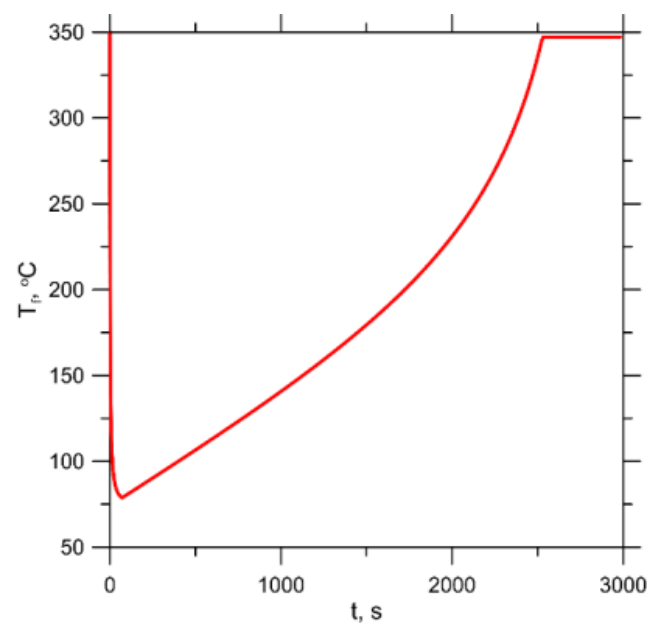

Fig. 6. The optimum fluid temperature during the first and second phase of drum heating; nominal fluid temperature in the drum $T_{\text {nom }}=347.1{ }^{\circ} \mathrm{C}$. 
The optimum temperature changes of the fluid in the first and second phase of heating are shown in Fig. 6.

Using exact analytical or numerical methods, it would be difficult to determine the temperature distributions shown in Fig. 5 because of the difficulty in obtaining a correct solution in the vicinity of $F_{O}=0$.

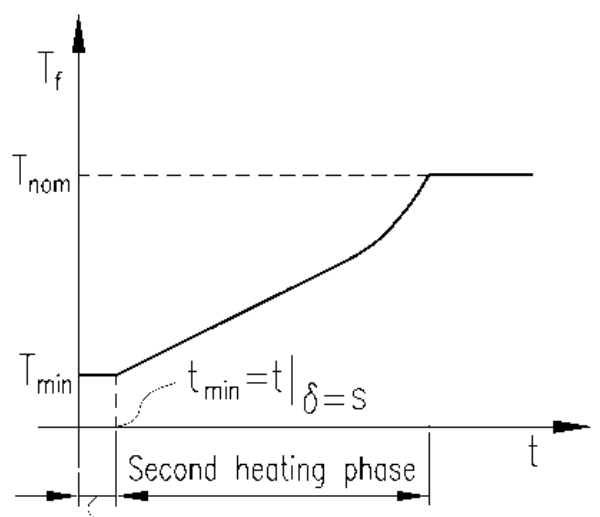

Initial heoting pose

Fig. 7. Simplified optimum time variations of the fluid temperature.

The stress due to the pressure was zero because $p=p_{a}$. The optimum fluid temperature drops very quickly to reach the minimum temperature $T_{f}=78.77^{\circ} \mathrm{C}$ at the end of the first phase of heating. The rapid reduction of the fluid temperature in the first phase of heating the pressure element is difficult to achieve in practice.

Therefore, the temperature changes in the first phase were simplified. It has been assumed that the temperature of the fluid changes stepwise from the initial temperature $T_{0}$ to the minimum temperature $T_{\min }$ (Fig. 7).

\section{Simulation of optimum heating of the drum-downcomer junction by the FEM using the ANSYS program}

The fluid temperature variation shown in Fig. 7 was used for calculations with ANSYS. The step-changing temperature of the fluid from $T_{0}$ to $T_{\min }$ can be easily realized in practice by quickly flooding the drum with hot water at $T_{\min }$ temperature.

The circumferential stress at point $P_{1}$ (Fig. 1) is the sum of the thermal stress and the stress caused by the pressure. Analysis of the results in Fig. 8 shows that after approximately 250 seconds of heating, the stress at point $P_{1}$ is close to the allowable stress. Up to a time of about 1250 seconds, the stress at point $P_{1}$ is close to the allowable stress. When the drum is heated further, the stress at point $P_{1}$ is reduced and significantly less than the permissible stress during heating. In the range of fluid temperatures higher than $160^{\circ} \mathrm{C}$, the increase in fluid temperature is accompanied by a rapid increase in pressure. Since the stress concentration factor for the stress due to the pressure at point $P_{1}$ is equal to 2.67 , the stress from the pressure with excess compensates thermal stress. This means that at an appropriately high pressure of more than $1.6 \mathrm{MPa}$, the permissible heating rate increases very quickly. With a pressure increase of up to about $4 \mathrm{MPa}$, the compensating effect of the pressure stress is so high that the fluid temperature can be increased at any rate.

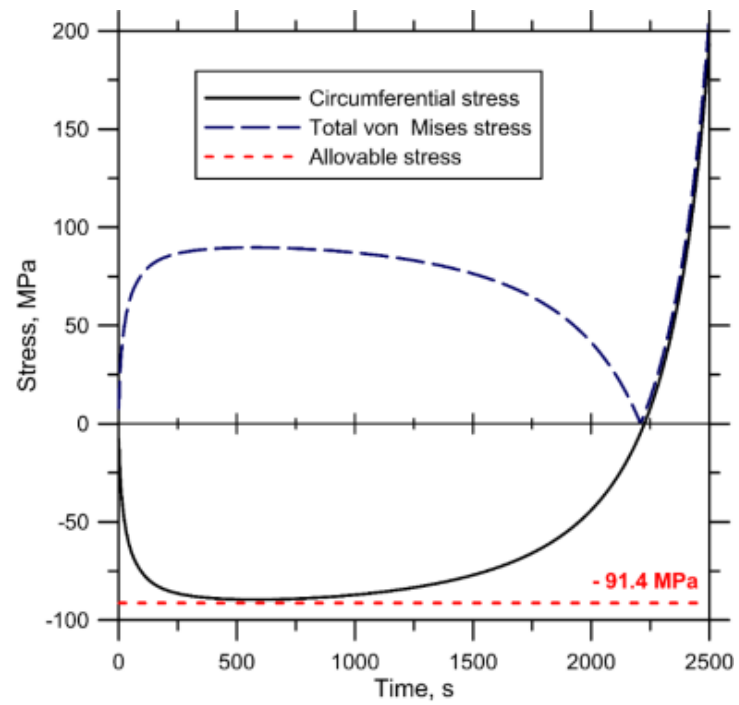

Fig. 8. Circumferential and Huber- von Mises equivalent stress at the point $P_{1}$.

The calculated time of the fluid temperature rise to the nominal temperature of $347.1^{\circ} \mathrm{C}$ is very short and amounts to about 2532 seconds. The heating time of the drum from the temperature of $20^{\circ} \mathrm{C}$ to $347.1^{\circ} \mathrm{C}$, according to the manufacturer's recommendations to heat the drum with the rate of $1 \mathrm{~K} / \mathrm{min}$ would be 19,620 seconds, i.e., 5 hours and 45 minutes. By heating the drum by the proposed method, the drum heating time is shortened by 17,088 seconds, i.e., by about 284 minutes. Figures 9 and 10 illustrate the distribution of temperature and equivalent Huber-Von Mises stress in the longitudinal section of the drumdowncomer connection at a time point of 750 seconds, respectively.

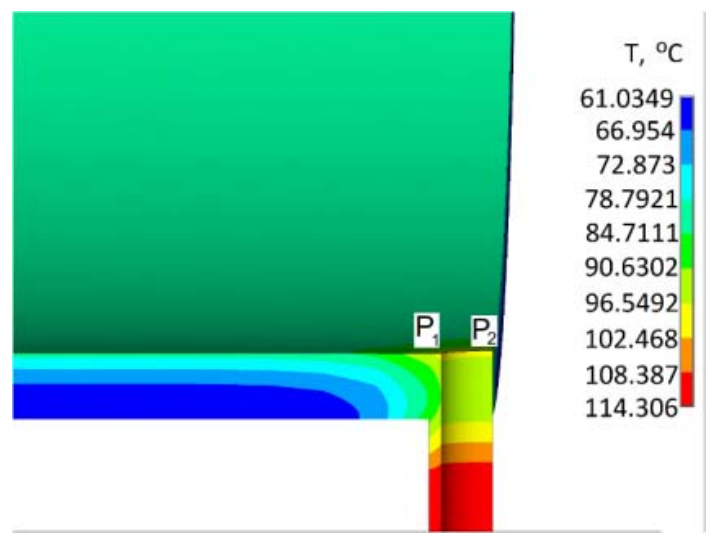

Fig. 9. Temperature distribution in the drum-downcomer connection at a time point of 750 seconds. 


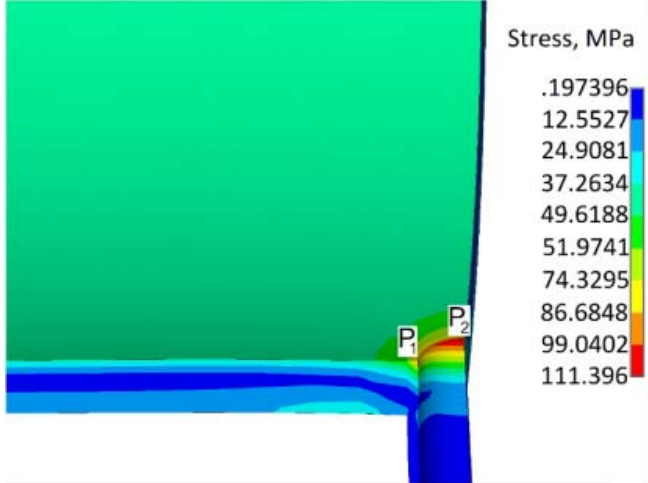

Fig. 10. Equivalent Huber-von Mises stress distribution in the drum-downcomer connection at a time point of 750 seconds.

The highest temperature is in the downcomer (connection tube) due to its much smaller thickness compared to the wall thickness of the drum. High temperature can also be observed on the inner surface of the drum near the edge of the hole, especially at point $P_{1}$. The highest equivalent stress at the edge of the hole occurs at point $P_{2}$ and lower at point $P_{1}$. At point $P_{1}$, the equivalent stress is lower because the circumferential thermal stress at point $P_{1}$ is compensated to a greater extent by the circumferential stress due to the pressure compared to point $P_{2}$. It should be noted that the optimum fluid temperature variations determined due to the total circumferential stresses at points $P_{1}$ and $P_{2}$ are similar, because at the same preset number of cycles the heating-cooling of the drum, the stresses permitted at points $P_{1}$ and $P_{2}$ are different.

\section{Conclusions}

The presented method of determining the optimum fluid temperature variations when heating cylindrical pressure elements weakened by holes can be easily applied in practice. In contrast to the European standard EN129523 , it is permissible to increase step-wise the temperature of the fluid at the beginning of the heating process of the pressure element. By optimization of heating of thickwalled pressure components of the boiler, the time of starting the boiler from a cold state can be significantly shortened. The example of optimum heating of the drum of the steam boiler with natural circulation shows that the heating time of the drum from ambient temperature to design temperature can be shortened more than seven times in comparison with the start-up time determined according to the recommendation of the boiler manufacturer.

\section{References}

1. A. Benato, A. Stoppato, S. Bracco, Energ Convers Manage 87, 1269-1280 (2014)

2. P. U. Sunil, J. Barve, P. S. V. Nataraj, Energy 126, 312-325 (2017).
3. J. Taler, P. Dzierwa, M. Jaremkiewicz, D. Taler, K. Kaczmarski, M. Trojan, T. Sobota, Energy 175, 645-666 (2019)

4. M. Jaremkiewicz, P. Dzierwa, D. Taler, J. Taler, Energy 175, 139-150 (2019)

5. J. Taler, P. Dzierwa, D. Taler, M. Jaremkiewicz, M. Trojan, Monitoring of Thermal Stresses and Heating Optimization Including Industrial Applications (Nova Science Publishers, Inc., New York, 2016)

6. D. Taler, J. Taler, Int J Heat Mass Tran 52, 2335 2342 (2019)

7. J. Taler, P. Dzierwa, D. Taler, P. Harchut, Energy 92, 160-170 (2015)

8. P. Dzierwa, D. Taler, M. Trojan, J. Taler, Heat Transfer Eng 39, 1217-1226 (2018)

9. H. Ribera, T.G. Myers, M.M. MacDevette, Appl Math Comput 354, 216-231 (2019)

10. S.L. Mitchell, T.G. Myers, Int J Heat Mass Tran 53, 3540-3551 (2010)

11. L. Prandtl, Zamm-Z Angew Math Me 18, 77-82 (1938)

12. K.F. Riley, M.P. Hobson, S.J. Bence, Mathematical Methods for Physics and Engineering (Cambridge University Press, Cambridge, 2006)

13. I.N. Bronstein, K.A. Semendjajev, Handbook of Mathematics (Springer, Berlin, 1985)

14. European Standard EN 12952-3, Water-tube boilers, and auxiliary installations. Part 3: Design and calculation for pressure parts (European Committee for Standardization, Brussels, 2001)

15. H.J. Kretzschmar, W. Wagner, International Steam Tables. Properties of Water and Steam based on the Industrial Formulation IAPWS-IF97 (Springer, Berlin, 2019) 